\title{
Hippocampal volume and 2-year outcome in depression
}

Klaus-Thomas Kronmüller, Johannes Pantel, Sebastian Köhler, Daniela Victor,

Frederik Giesel, Vincent A. Magnotta, Christoph Mundt, Marco Essig and Johannes Schröder

\section{Summary}

Although the hippocampus has been found to be smaller in people with depression, the clinical relevance of this is unclear. We investigated hippocampal volume (using highresolution magnetic resonance imaging) and 2-year outcome in 57 patients with major depression. The left and right hippocampal volumes of patients with a depression relapse were significantly smaller than those of healthy controls. Our results support the hypothesis that the hippocampus is crucial in the outcome of depression.

\section{Declaration of interest}

None.
Studies have found abnormalities in hippocampal volumes in patients with major depression. ${ }^{1,2}$ Although the hippocampus has been found to be smaller in patients with depression, prospective longitudinal studies are necessary to investigate whether a smaller hippocampal volume predicts the course of depression. The only study that investigated the predictive effect in a longer follow-up period reported that patients who had not remitted at the 1-year follow-up investigation showed significantly reduced left and right hippocampal volumes compared with patients who had remitted; however, no relapse prediction was done in this study. ${ }^{3}$ In recent meta-analysis, gender differences in hippocampal volumes were discussed. ${ }^{1}$ The aim of the present study was to examine the relationship between hippocampal volume and 2-year outcome of depression. The hypothesis was that patients with recurring depression have smaller hippocampal volumes at the beginning of the study in comparison with patients with stable remission, and healthy controls. Additionally, gender effects were examined.

\section{Method}

A total of 57 in-patients with DSM-IV major depression treated in the Department of Psychiatry of the University in Heidelberg were recruited. ${ }^{4}$ Diagnoses were based on the Structured Clinical Interview for DSM-IV (SCID). The mean age of the 33 female and 24 male patients at initial assessment was 43.54 years (s.d. $=12.82$, range 18-64). Twenty-seven (47.37\%) were married, 22 (38.6\%) had a high and $35(61.4 \%)$ a low level of school education; 26 $(45.61 \%)$ patients had a first episode of major depression whereas $31(54.39 \%)$ had recurrent depression. Mean score on the Hamilton Rating Scale for Depression (HRSD) at admission to treatment was 22.74 (s.d.=6.58). For comparison, 30 healthy participants without a history of psychiatric disorders according to the SCID were recruited. Patients with major depression did not differ significantly from healthy controls regarding age, gender, height, weight, handedness, social class, education or alcohol consumption.

The main investigations were carried out at the beginning of treatment, at discharge from hospital as well as 1 and 2 years after discharge. Relapse was defined according to DSM-IV criteria of major depression. Follow-up criteria of Frank et $a l^{5}$ were used in combination with the Longitudinal Interval Follow-up Evaluation (LIFE). ${ }^{6}$ After a complete description of the study was given to patients and healthy controls, written informed consent was obtained. The study protocol was approved by the local ethics committee and was prepared in accordance with the Declaration of Helsinki.

Magnetic resonance imaging (MRI) scans of the whole brain were obtained by using a Siemens $1.5 \mathrm{~T}$ magnetic resonance scanner. $\mathrm{T}_{1}$-weighted three-dimensional magnetisation-prepared rapid gradient echo sequences (3D-MPRAGE) and $\mathrm{T}_{2}$-weighted images were acquired. ${ }^{4}$ Image processing was performed on a computer workstation using BRAINS (Brain Research: Analysis of Images, Networks, and Systems) software. As part of the segmentation procedure of BRAINS, intracranial volume and total brain volume were determined semi-automatically. Hippocampal volume was measured by using a reliable and validated method, previously described in detail. ${ }^{7}$ The hippocampal formation was traced manually on the continuous segmented image (stereo image) provided by BRAINS. A detailed description of boundary definition is provided at http://iowa-mhcrc.psychiatry.uiowa.edu/mhcrc/ IPLpages/manual_tracing. htm. The intraclass correlation coefficient (ICC) was 0.97 for the left hippocampus and 0.98 for the right hippocampus.

Morphometric data were normally distributed. We performed ANOVAs with repeated measurement to examine the relationship between baseline and follow-up variables. The main interaction effects of the within-participants factor time and the betweenparticipant factors outcome and gender were assessed by using total cranial volume and age as the cofactors. Significant interactions were resolved by univariate ANCOVAs on the hippocampal volumes for each region and prognostic group, controlling for total cranial volume and age. Contrasts were performed using the Tukey test. Results were considered statistically significant if at or below $5 \%$ probability (two-tailed). Analyses were performed with SAS Version 9.12 for Windows.

\section{Results}

Follow-up data were obtained for all 57 patients. In the follow-up period of 2 years, $49(85.96 \%)$ of the 57 patients had remitted from index depression: 21 (36.84\%) patients suffered a recurrence after recovery on average after 47.95 weeks (s.d.=29.13). Because of the small number of patients with a chronic course and because we do not know whether these patients will recover or have recurrences in the future, we excluded this group from the analyses.

The hippocampal volumetric data are shown in Table 1. A significant main effect on hippocampal volume was found for outcome $(F=4.93$, d.f. $=2,71, P=0.01)$. The main gender effect 
Table 1 Hippocampal volumes of patients with and without recurrence of depression compared with healthy controls: 2-year

follow-up

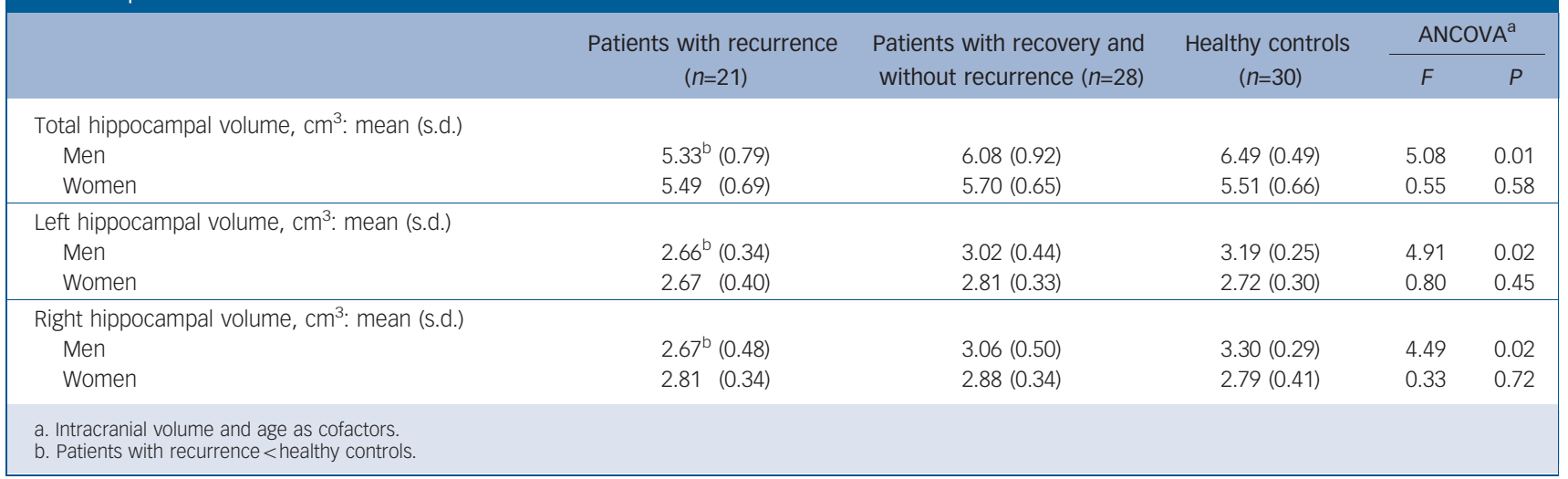

was not significant $(F=3.91$, d.f. $=1,71, P=0.05)$, whereas the interaction between outcome and gender was significant $(F=3.38$, d.f.=2,71, $P=0.04$ ). The hemisphere (left-right asymmetry) main effect $(F=0.22$, d.f. $=1,71, P=0.64)$ and the interaction effect of hemisphere and outcome $(F=0.16$, d.f. $=2,71, P=0.85)$ were both not significant. The covariates age and intracranial volume were not significant either. Post hoc univariate ANCOVA revealed that male patients with a recurrence of depression had significantly smaller left $(F=4.91$, d.f. $=2,24, P=0.02)$, right $(F=4.49$, d.f. $=2,24$, $P=0.02)$ and total $(F=5.08$, d.f. $=2,24, P=0.01)$ hippocampal volumes than healthy male controls. No significant differences of left, right and total hippocampal volumes were found for women. Additionally, there was no significant difference in the total brain volume between the groups. Even after controlling for current depression, duration of illness, age at onset, number of episodes, severity of illness and medication using ANCOVA, the differences in hippocampal volumes between the two outcome groups remained significant.

\section{Discussion}

Overall, 37\% of patients had a recurrence in the 2-year follow-up period, which is in accordance with most follow-up studies on depression. In the present study, it could be shown for the first time that male patients with a recurrence after recovery from depression had a significantly smaller left and right hippocampal volume in comparison with healthy controls. No significant leftright asymmetry effects for outcome prediction were revealed. Since no significant differences in total brain volume could be found between the groups, and intracranial volume and age were controlled as covariates, the effect of hippocampal volume change can be regarded as being specific and cannot be attributed to general brain morphological changes or age effects. Thus, we could complement the results of Frodl et $a l^{3}$ who showed a bilateral hippocampal reduction for patients who had not remitted.

Regarding gender effects, results of past studies are heterogeneous. ${ }^{1,2}$ However, it is well known that the hippocampal volume of healthy men is greater than that of healthy women and that it decreases over the lifespan only in men. Some recent studies found smaller hippocampal volumes more often in men with depression than in women with depression. ${ }^{8}$ Thus, it can be assumed that hippocampal volume reduction is a genderspecific factor in the pathophysiology of depression.

It remains unclear whether smaller hippocampal volumes exist before the onset of depression or the reductions are the result of depression or other psychosocial influences. Analysis of the prognostic effects of hippocampal volumes is no substitute for longitudinal structural imaging studies in obtaining more information on the causality of the relationship. Our results strongly point to the clinical relevance of morphological alterations of the hippocampus by showing that these changes predict relapse in male patients. Moreover, the present findings can also contribute to the reformulation of neuroanatomical models of the pathophysiology of depression in a more gender-specific conceptualisation. In future studies, the mechanisms behind changes in hippocampal volume and their relationship to treatment and outcome of depression should be addressed.

Klaus-Thomas Kronmüller, MD, Department of Psychiatry, University of Heidelberg Johannes Pantel, MD, Department of Psychiatry and Psychotherapy, University of Frankfurt; Sebastian Köhler, MD, Daniela Victor, PhD, Department of Psychiatry, University of Heidelberg; Frederik Giesel, MD, Department of Radiology, German Cancer Research Centre, Heidelberg; Vincent A. Magnotta, PhD, Department of Psychiatry, University of lowa; Christoph Mundt, MD, Department of Psychiatry, University of Heidelberg; Marco Essig, MD, Department of Radiology, German Cancer Research Centre, Heidelberg; Johannes Schröder, MD, Department of Psychiatry, University of Heidelberg

Correspondence: Klaus-Thomas Kronmüller, Department of Psychiatry, University of Heidelberg, Voßstraße 4, Heidelberg 69115, Germany. Email: klaus_kronmueller@med.uni-heidelberg.de

First received 14 May 2007, final revision 17 Dec 2007, accepted 20 Feb 2008

\section{Acknowledgements}

Supported by the German Federal Research Ministry within the German Research Networks in Medicine promotion as part of the German Research Network on Depression project.

\section{References}

1 Videbech P, Ravnkilde B. Hippocampal volume and depression. A metaanalysis of MRI studies. Am J Psychiatry 2004; 161: 1957-66.

2 Campbell S, Marriott M, Nahmias C, MacQueen GM. Lower hippocampal volume in patients suffering from depression: a meta-analysis. Am J Psychiatry 2004; 161: 598-607.

3 Frodl $T$, Meisenzahl EM, Zetzsche $T$, Höhne $T$, Banac $S$, Schorr $C$, Jäger $M$, Leinsinger $G$, Bottlender R, Reiser M, Möller HJ. Hippocampal and amygdala changes in patients with major depressive disorder and healthy controls during a 1-year follow-up. J Clin Psychiatry 2004; 65: 492-9.

4 Kronmüller KT, Pantel J, Götz B, Köhler S, victor D, Mundt C, Magnotta VA, Giesel F, Essig M, Schröder J. Life events and hippocampal volume in firstepisode major depression. J Affect Disord 2008; doi 10.1016/j.jad.2008.01.022.

5 Frank E, Prien RF, Jarrett RB, Keller MB, Kupfer DJ, Lavori PW, Rush AJ, Weissman MM. Conceptualization and rationale for consensus definitions of terms in major depressive disorder. Remission, recovery, relapse and recurrence. Arch Gen Psychiatry 1991; 48: 851-5.

6 Keller MB, Lavori PW, Friedman B, Nielsen E, Endicott J, McDonald-Scott P, Andreasen NC. The Longitudinal Interval Follow-up Evaluation. A comprehensive method for assessing outcome in prospective longitudinal studies. Arch Gen Psychiatry 1987; 44: 540-8.

7 Pantel J, O'Leary DS, Cretsinger K. A new method for the in vivo volumetric measurement of the human hippocampus with high neuroanatomical accuracy. Hippocampus 2000; 10: 752-8.

8 Frodl T, Meisenzahl EM, Zetzsche T. Hippocampal changes in patients with a first episode of major depression. Am J Psychiatry 2002; 159: 1112-8. 